HIP-1999-78/TH

PPD-IOP-99/25

\title{
Large hierarchy from extra dimensions
}

\author{
Masud Chaichian ${ }^{1}$ \\ Department of Physics, High Energy Physics Division, University of Helsinki and \\ Helsinki Institute of Physics, P.O. Box 9 (Siltavuorenpenger 20 C), \\ FIN-00014 Helsinki, Finland
}

Archil B. Kobakhidze ${ }^{2}$

Andronikashvili Institute of Physics, Tamarashvili str. 6, GE-380077 Tbilisi, Georgia

\begin{abstract}
We argue that the familiar gauge hierarchy between the fundamental Planck scale $M_{P l}$ and the electroweak scale $M_{W}$, can be naturally explained in higher dimensional theories with relatively large radii $\left(R_{c}>1 / M_{P l}\right)$ extra dimensions. In particular, we show that it is possible that the electroweak Higgs mass at high energies is of the order of $M_{P l}$, but radiative corrections drive it to an infrared stable fixed-point $\sim M_{W}$ at low energies thus inducing a large hierarchy without any fine tuning of parameters.
\end{abstract}

1 E-mail: Masud.Chaichian@helsinki.fi. Supported by the Academy of Finland under the Project No. 163394.

2 E-mail: achiko@iph.hepi.edu.ge. Partially supported by the Grant No. 2-10 of the Georgian Academy of Sciences, by the Georgian Young Scientists Presidential Award and by the INTAS Grant 96-155. 
Despite the fact that the Standard Model (SM) of strong and electroweak interactions successfully describes all observed phenomena at currently accessible energies $M_{W} \sim 100 \mathrm{GeV}$, there are several indications for new physics beyond the SM to exist. From the theoretical point of view, perhaps the most strict hint for such new physics is the gauge hierarchy problem which deals with the natural explanation not only of the hierarchy of scales, $M_{W} / \Lambda \ll 1$, but also of the stability of this hierarchy under the quantum corrections, where the SM is regarded as a low-energy limit of a certain more fundamental theory with characteristic scale $\Lambda$ ( $\Lambda$ can be, say, the grand unified scale $M_{G U T} \sim 10^{16}$ $\mathrm{GeV}$, or the Planck scale $\left.M_{P l} \approx 10^{18} \mathrm{GeV}\right)$. The solution to this problem in four dimensions has been usually attributed to supersymmetric models, or models with dynamical gauge symmetry breaking with characteristic scales of the order of $\mathrm{TeV}$.

On the other hand, it is widely believed that a unified theory of all fundamental forces in nature (including gravity) can be consistently constructed in space-time with more than four dimensions. That is why the novel attack to the gauge hierarchy problem within the higher dimensional theories, much motivated by studies of non-perturbative superstring and M-theories, has recently received considerable attention. It has been proposed in [1] that the fundamental Planck scale can be as low as few $\mathrm{TeV}$, requiring the presence of two extra compact dimensions at least with sub-millimeter radii, $R_{c}=\frac{1}{\mu_{c}} \sim 1$ $\mathrm{mm}$, and that SM particles and forces are confined on a 3-brane in the extra dimensions in order to avoid the conflict with presently accessible experiments. However, while this scenario does eliminate the hierarchy between the weak $M_{W}$ and Planck scale $M_{P l}$, it introduces a new hierarchy between $\mu_{c}$ and $M_{W}$ $\left(\frac{\mu_{c}}{M_{W}} \approx \frac{M_{W}}{M_{P l}}\right)$ and stability of large extra dimensions also remains as a critical question. In this connection, another distinct higher dimensional scenario proposed in [2] provides an exponential hierarchy between the weak $M_{W}$ and Planck $M_{P l}$ scales through the special solutions for the five-dimensional Einstein equation with the cosmological constant and 3-branes involving "warp" factor in the metric tensor $[3][$. Then the required hierarchy is naturally generated even with small extra dimensions $\left(\frac{\mu_{c}}{M_{P l}} \approx 10^{-2}\right)$, so the model easily avoids current constraints from particle physics [5], astrophysics and cosmology [6] Several studies of rich phenomenological and cosmological consequences of such a scenario have been proposed recently [9]. Note, however, that a common deficiency of the presented models is the ad hoc fine tunings

\footnotetext{
3 A remarkable thing offered by such a solutions is the possibility to reproduce the four-dimensional Newton's law in our universe even with infinitely large (noncompact) extra dimensions. However, to solve the gauge hierarchy problem in this case more complex constructions are required [4].

4 It has been emphasized in [7] the troubles with cosmological expansion in the scenario where the SM is placed on the 3 -brane with negative tension [2]. Also, the difficulty to reproduce gauge coupling unification is pointed out in [8].
} 
required between the cosmological constants in the bulk and on the 3 -branes, in order to obtain the desired solution to the Einstein equations. The stability of such fine tuning seems also to be problematic.

In this paper we suggest a new mechanism for gauge hierarchy in higher dimensional theories with relatively large radii compact extra dimensions. We explore the old idea that the large hierarchy of scales can be explained without any fine tuning of parameters if the electroweak Higgs boson anomalous mass dimension is $A \geq 2[10]$ D. However, $A \geq 2$ is highly undesirable, since anomalous mass dimension $A$, being proportional to coupling constants, is usually $\ll 1$, unless some of the couplings (Higgs self-interacting coupling or/and Yukawa couplings) are non-perturbative below the scale $\Lambda$, or there is an unrealistically large number of degrees of freedom ensuring $A \geq 2$ [10]. Needless to say, that it is very difficult (if ever possible) to construct a realistic model obeying such conditions. In higher dimensional theories, however, the situation is drastically changed. The point is that, owing to the power-law (in contrast to the logarithmic in four dimensions) evolution of the theory parameters, the Higgs vacuum expectation value (VEV), while being of the order of $\Lambda$ at high energies, rapidly decrease down to the infrared stable fixed point $\sim M_{W}$ even for the small values of $A$, thus naturally inducing a large hierarchy even in the case of SM with the ordinary number of colours and flavours. Let us demonstrate this phenomenon explicitly on the simplified example of the $S U(N)$-symmetric Higgs-Yukawa system with $N_{c}$ colours.

Our starting action in $D=4+\delta$ dimensions ( $\delta$ is the number of extra compact dimensions) is

$$
\begin{aligned}
S_{\Lambda_{0}}= & \int d^{4+\delta}\left[Z\left(\Lambda_{0}\right) \partial_{\mu} \phi^{+} \partial^{\mu} \phi-\mu^{2}\left(\Lambda_{0}\right) \phi^{+} \phi+\frac{1}{2} \lambda\left(\Lambda_{0}\right)\left(\phi^{+} \phi\right)^{2}\right. \\
& +Z_{L}\left(\Lambda_{0}\right) \bar{\psi}_{L} i \gamma_{\mu} \partial^{\mu} \psi_{L}+Z_{R}\left(\Lambda_{0}\right) \bar{\psi}_{R} i \gamma_{\mu} \partial^{\mu} \psi_{R} \\
& \left.+\left(h\left(\Lambda_{0}\right) \bar{\psi}_{L} \phi \psi_{R}+\text { h.c. }\right)\right],
\end{aligned}
$$

where $\phi$ is an $N$-component complex scalar field, $\psi_{L}$ is an $N$-component lefthanded fermion field with $N_{c}$ colours and $\psi_{R}$ is a right-handed $S U(N / 2)-$ singlet fermion with $N_{c}$ colours again. $Z, Z_{L}$, and $Z_{R}$ in (1) are the field renormalization factors which we choose to be equal to 1 at the scale $\Lambda_{0}$. In the case of $N=2$ and $N_{c}=3$ the action (1) is just the SM action in the limit of vanishing gauge couplings and Higgs-Yukawa couplings except for one type of quarks.

Theory with action (1) in higher dimensions $(\delta \neq 0)$ is known to be nonrenormalizable, but it can be well defined by introducing an ultraviolet cut-off $\Lambda_{0}$, which is natural to identify with the fundamental Planck scale $M_{P l}$. At

5 Somewhat similar idea have been proposed in [11] to study the patterns of GUT symmetry breaking. 
low energies one can consistently describe the theory using Wilsonian effective action approach $[12]^{\square}$. The basic idea behind this approach is first to integrate out momentum modes between a cut-off scale $\Lambda_{0}$ and lower energy scale $\Lambda$, rather than to integrate over all momentum modes in one go. The remaining integral from $\Lambda$ to zero may again be expressed as a partition function, but the bare action $S_{\Lambda_{0}}(1)$ is replaced by a complicated effective action $S_{\Lambda}$ (Wilsonian effective action) and the overall cut-off $\Lambda_{0}$ by the effective cut-off $\Lambda$, in such a way that all physics, i.e. Green functions, are left invariant. The difference in $S_{\Lambda}$ induced by the change of the cut-off is determined integrating "shell modes" with momenta between $\Lambda$ and $\Lambda+\delta \Lambda$ and for an infinitesimal $\delta \Lambda$ becomes a Gaussian path integral which can be exactly carried out. Thus, the scale dependence of the Wilsonian effective action is given by the exact functional differential equation

$$
\Lambda \frac{\partial S_{\Lambda}}{\partial \Lambda}=\mathcal{O}\left[S_{\Lambda}\right]
$$

where $\mathcal{O}\left[S_{\Lambda}\right]$ is a non-linear operator acting on the functional $S_{\Lambda}$. However, for the practical calculations it is inevitable to approximate the evolution equation (2). Usually, one expands the effective action $S_{\Lambda}$ in terms of the number of derivatives included in the general operators. In the first order of this approximation any derivative couplings are dropped except for the kinetic term. This is the so-called local potential approximation (LPA) [14], which we adopt here. In the "sharp cut-off" scheme we obtain the renormalization group (RG) equation for the local effective scalar potential (Wegner-Houghton equation):

$$
\begin{aligned}
\Lambda \frac{\partial V}{\partial \Lambda}= & -\frac{K_{D}}{2} \Lambda^{D}\left[\ln \left(Z \Lambda^{2}+V^{\prime}(\rho)+2 \rho V^{\prime \prime}(\rho)\right)+\right. \\
& \left.(2 N-1) \ln \left(Z \Lambda^{2}+V^{\prime}(\rho)\right)-2^{\frac{D}{2}} N_{c} \ln \left(Z_{L} Z_{R} \Lambda^{2}+h^{2} \rho\right)\right],
\end{aligned}
$$

where $\rho=\frac{1}{2}|\phi|^{2}$, prime denotes the derivative with respect to $\rho$ and $K_{D}$ is the $D$-dimensional angular integral

$$
K_{D}=\frac{2^{1-D}}{\pi^{-D / 2} \Gamma(D / 2)}
$$

( $\Gamma$ is the Euler function). Now, since we are interested in the spontaneously broken regime, let us expand the effective potential $V$ around its minimum $\rho_{0}(\Lambda)$ (commoving scheme) [15]:

$$
V(\rho)=u_{0}(\Lambda)+u_{1}(\Lambda)\left(\rho-\rho_{0}(\Lambda)\right)+\frac{1}{2} u_{2}(\Lambda)\left(\rho-\rho_{0}(\Lambda)\right)^{2}+\ldots
$$

6 Recently, this approach have been applied to the higher dimensional theories in [13]. 
where the minimum $\rho_{0}(\Lambda)$ is defined from the extremum equation

$$
\left.\frac{\partial V}{\partial \rho}\right|_{\rho=\rho_{0}}=0 .
$$

Taking the total $\ln \Lambda$-derivative of (6), we obtain RG equation for $\rho_{0}(\Lambda)$ :

$$
\begin{aligned}
\Lambda \frac{d \rho_{0}}{d \Lambda}= & \frac{K_{D}}{2} \Lambda^{D}\left[\frac{3}{Z \Lambda^{2}+2 \rho_{0} \lambda}+(2 N-1) \frac{1}{Z \Lambda^{2}}-\right. \\
& \left.2^{\frac{D}{2}} N_{c} \frac{h^{2}}{\lambda} \frac{1}{Z_{L} Z_{R} \Lambda^{2}+h^{2} \rho_{0}}\right],
\end{aligned}
$$

where we have denoted the scalar quadric coupling as $\lambda(\Lambda) \equiv u_{2}(\Lambda)=$ $\left.\frac{\partial^{2} V}{\partial \rho^{2}}\right|_{\rho=\rho_{0}}$. By substituting (5) into (3), it will be also easy to obtain an infinite set of coupled RG equations for $u_{i}(\Lambda)=\left.\frac{\partial^{i} V}{\partial \rho^{i}}\right|_{\rho=\rho_{0}}(i=3,4, \ldots)$ couplings,

$$
\Lambda \frac{d u_{i}}{d \Lambda}=u_{i+1} \Lambda \frac{d \rho_{0}}{d \Lambda}+\left.\frac{\partial^{i}\left(\Lambda \frac{\partial V}{\partial \Lambda}\right)}{\partial \rho^{i}}\right|_{\rho=\rho_{0}} .
$$

Thus, the Wilson RG framework allows, in principle, to take into account higher-dimensional operators in the evolution of couplings in (5). However, we restrict ourself to consider only the potential in the vicinity of $\rho_{0}(\Lambda)$ approximated by a $\phi^{4}$-potential. This corresponds to the truncation

$$
u_{3}=u_{4}=\ldots=0 \text {. }
$$

In this approximation the evolution equation for the scalar quadric coupling $\lambda(\Lambda)$ looks as

$$
\begin{aligned}
\Lambda \frac{d \lambda}{d \Lambda}= & \frac{K_{D}}{2} \Lambda^{D}\left[\frac{9 \lambda^{2}}{\left(Z \Lambda^{2}+2 \rho_{0} \lambda\right)^{2}}+(2 N-1) \frac{\lambda^{2}}{Z^{2} \Lambda^{4}}-\right. \\
& \left.2^{\frac{D}{2}} N_{c} \frac{h^{4}}{\left(Z_{L} Z_{R} \Lambda^{2}+h^{2} \rho_{0}\right)^{2}}\right] .
\end{aligned}
$$

Now, let us define the renormalized quantities

$$
\begin{aligned}
& \rho_{R}=Z \rho_{0}, \\
& \lambda_{R}=Z^{-2} \lambda, \\
& h_{R}^{2}=Z_{L}^{-1} Z_{R}^{-1} Z^{-1} h^{2},
\end{aligned}
$$

and the anomalous dimensions

$$
\gamma=-\Lambda \frac{d \ln Z}{d \Lambda}
$$




$$
\begin{aligned}
\gamma_{L} & =-\Lambda \frac{d \ln Z_{L}}{d \Lambda}, \\
\gamma_{R} & =-\Lambda \frac{d \ln Z_{R}}{d \Lambda}
\end{aligned}
$$

The anomalous dimensions (12) had been calculated in [16] in the smooth cut-off approximation (average action), which results we adopt here:

$$
\begin{aligned}
\gamma & =\frac{K_{D}}{D} \Lambda^{D-4} 2^{\frac{D}{2}} N_{c} h_{R}^{2} \\
\gamma_{L} & =\frac{2 K_{D}}{D} \Lambda^{D-4} 2^{\frac{D}{2}} h_{R}^{2} \\
\gamma_{R} & =\frac{2 K_{D}}{D} \Lambda^{D-4} 2^{\frac{D}{2}} N h_{R}^{2}
\end{aligned}
$$

Then, from Eqs. (7) and (10)-(13) the RG equations for renormalized parameters $\rho_{R}$ and $\lambda_{R}$ can be easily obtained,

$$
\Lambda \frac{d \rho_{R}}{d \Lambda}=\frac{K_{D}}{2}\left((2 N+2)-2^{\frac{D}{2}} N_{c} \frac{h_{R}^{2}}{\lambda_{R}}\right) \Lambda^{D-2}+A_{\delta} \rho_{R},
$$

with

$$
A_{\delta}=-\frac{K_{D}}{2} \Lambda^{D-4}\left[6 \lambda_{R}-2^{\frac{D}{2}} N_{c} \frac{h_{R}^{4}}{\lambda_{R}}\right]-\gamma
$$

and

$$
\Lambda \frac{d \lambda_{R}}{d \Lambda}=\frac{K_{D}}{2} \Lambda^{D-4}\left[(2 N+8) \lambda_{R}^{2}-2^{\frac{D}{2}} N_{c} h_{R}^{4}\right]+2 \gamma \lambda_{R}
$$

where we have expanded the right-hand sides of equations (14) and (16) in powers of $\frac{2 \rho_{R} \lambda_{R}}{\Lambda^{2}}$ and $\frac{h_{R}^{2} \rho_{R}}{\Lambda^{2}}$ and have kept only the leading terms $O\left(\rho_{R}\right)$ in (14) and $O\left(\lambda_{R}^{2}\right)$ and $O\left(h_{R}^{4}\right)$ in (16). This approximation is valid if

$$
\begin{aligned}
\frac{2 \rho_{R} \lambda_{R}}{\Lambda^{2}} & \ll 1, \\
\frac{h_{R}^{2} \rho_{R}}{\Lambda^{2}} & \ll 1,
\end{aligned}
$$

and, as we will show below, correctly reproduce the results of the standard one-loop perturbation theory. Actually, the relations $2 \rho_{R} \lambda_{R}\left(m_{S}\right)=m_{S}^{2}$ and $h_{R}^{2} \rho_{R}\left(m_{F}\right)=m_{F}^{2}$ determine the physical masses of the scalar radial mode and the fermion field, respectively, and thus below the scales $m_{S}$ and $m_{F}$ scalar and fermion fields become decoupled correspondingly.

$A_{\delta}$ of Eq. (15), which appears in the last term of (14) is just the anomalous dimension for the VEV of the scalar field. Clearly, $A_{\delta}$ has to be evaluated at 
the critical value $\rho_{R}^{c r}$, which is defined by solving (14) with first term only

$$
\rho_{R}^{c r}(\Lambda \rightarrow 0)=0
$$

The critical value $\rho_{R}^{c r}$ corresponds to the phase transition between the symmetric and spontaneously broken phases. Thus the anomalous $A_{\delta}$ has a simple physical interpretation [17]: It governs the scale dependence of the deviation of scalar field VEV from the critical value (18) resulting to the nonzero VEV for the scalar field

$$
\begin{aligned}
\left\langle\phi^{2}\right\rangle & =\lim _{\Lambda \rightarrow 0} \rho_{R}(\Lambda)=\lim _{\Lambda \rightarrow 0}\left(\rho_{R}^{c r}(\Lambda)+v_{R}^{2}(\Lambda)\right)=v^{2}, \\
\Lambda \frac{d v_{R}^{2}}{d \Lambda} & =A_{\delta} v_{R}^{2} .
\end{aligned}
$$

The evolution equation for the renormalized Yukawa coupling $h_{R}$ can be also derived from the effective action $S_{\Lambda}$ with nonzero fermion background fields $\psi$. Keeping the leading $O\left(h_{R}^{4}\right)$ terms one obtains

$$
\Lambda \frac{d h_{R}^{2}}{d \Lambda}=\left(\gamma+\gamma_{L}+\gamma_{R}\right) h_{R}^{2}
$$

It is obvious that the set of the RG equations (16), (20) and (21) is approximately valid for $\Lambda \gg \mu_{c}$ if some spatial dimensions are compactified. Here we simply ignore the threshold effects and assume that below the compactification scale $\mu_{c}$ the theory is four-dimensional, while above $\mu_{c}$ it is $D$-dimensional with flat (non-compact) $\delta$ extra dimensions. In $D$ dimensions one can define effective four-dimensional parameters by appropriately rescaling the fields and couplings:

$$
\begin{aligned}
\bar{v}^{2} & =\left(2 \pi R_{c}\right)^{\delta} v_{R}^{2}, \\
\bar{\lambda} & =\left(2 \pi R_{c}\right)^{-\delta} \lambda_{R}, \\
\bar{h} & =\left(2 \pi R_{c}\right)^{-\frac{\delta}{2}} h_{R} .
\end{aligned}
$$

For the four-dimensional renormalized parameters (22) we finally obtain the evolution equations:

$$
\begin{aligned}
& \Lambda \frac{d \bar{v}^{2}}{d \Lambda}=\frac{K_{D}}{2}\left(\frac{2 \pi \Lambda}{\mu_{c}}\right)^{\delta}\left[-6 \bar{\lambda}-\frac{2^{\frac{D}{2}+1}}{D} N_{c} \bar{h}^{2}+2^{\frac{D}{2}} N_{c} \frac{\bar{h}^{4}}{\bar{\lambda}}\right] \bar{v}^{2}, \\
& \Lambda \frac{d \bar{\lambda}}{d \Lambda}=\frac{K_{D}}{2}\left(\frac{2 \pi \Lambda}{\mu_{c}}\right)^{\delta}\left[(2 N+8) \bar{\lambda}^{2}+\frac{2^{\frac{D}{2}+2}}{D} N_{c} \bar{h}^{2} \bar{\lambda}-2^{\frac{D}{2}} N_{c} \bar{h}^{4}\right],
\end{aligned}
$$

7 Here we assume that $\delta=D-4$ extra dimensions are compactified on a circle of a fixed radius $R_{c}=\frac{1}{\mu_{c}}$. The factor $\left(2 \pi R_{c}\right)^{\delta}$ is just the volume of extra space appeared in the effective four-dimensional action after one integrates over the extra space. 


$$
\Lambda \frac{d \bar{h}^{2}}{d \Lambda}=\frac{K_{D}}{2}\left(\frac{2 \pi \Lambda}{\mu_{c}}\right)^{\delta} \frac{2(N+1)+2^{\frac{D}{2}} N_{c}}{D} \bar{h}^{4} .
$$

Note, that by taking $\delta=0$ the set of Eqs. (23a-c) correctly reproduces the familiar one-loop results of perturbation theory in four dimensions. The crucial role of the extra dimensions in solving the gauge hierarchy problem can be seen from Eqs. (23a-c) even without performing numerical calculations. Indeed, ignoring for the moment the running of $\bar{\lambda}$ and $\bar{h}$, one finds from (23a)

$$
\frac{\bar{v}\left(M_{W}\right)}{\bar{v}\left(M_{P l}\right)}=\left(\frac{M_{W}}{\mu_{c}}\right)^{\frac{\omega_{0}}{2}} \exp \left[\frac{(2 \pi)^{\delta}}{2 \delta} \omega_{\delta}\left(1-\left(\frac{M_{p l}}{\mu_{c}}\right)^{\delta}\right)\right]
$$

where $\omega_{\delta}=A_{\delta} /(\Lambda)^{\delta}$. The exponential factor in (24) can be naturally small in the case of extra dimensions $(\delta \neq 0)$ even for small (but positive) values of $\omega_{\delta}$, providing the desired hierarchy $\frac{\bar{v}\left(M_{W}\right)}{\bar{v}\left(M_{P l}\right)} \approx \frac{M_{W}}{M_{P l}}$, while in four dimensions this ratio is of the order of $O(1 \div 10)$ unless $\omega_{0}=A_{0} \gtrsim 2$, that can, however, never be obtained in perturbation theory since for small couplings $A_{0}$ is proportional to these couplings [10].

Of course, the actual solutions of the set of Eqs. (23a-c) is more complicated, since the Yukawa and self-interaction couplings also exhibit fast (power-law) running and the approximation of the constant $\bar{\lambda}$ and $\bar{h}$ is very crude. We have analyzed Eqs. (23) numerically. The Yukawa coupling $\bar{h}$ rapidly decreases going down in the energy region between $M_{P l}$ and $\mu_{c}$ and drives to the infrared stable fixed-point $\bar{h}=0$. If the Yukawa coupling dominates over the $\bar{\lambda}\left(\bar{h}^{2} \gg\right.$ $\bar{\lambda}) \bar{\gamma}$ then $\bar{\lambda}$ at the same time increases for smaller energies, until $\bar{\lambda}$ becomes large enough so that the terms proportional to $\bar{\lambda}^{2}$ and $\bar{h}^{2} \bar{\lambda}$ cancel the term proportional to $\bar{h}^{4}$ in $(23 \mathrm{~b})$. Thus, $\bar{\lambda}$ approaches the infrared stable fixedpoint, $\bar{\lambda} \sim \bar{h}^{2}$. At the same time, even starting with large initial values of $\bar{v}\left(M_{P l}\right) \lesssim M_{P l}, \bar{v}$ rapidly decreases and below the $\mu_{c}$ changes very slowly. Thus, for certain $\mu_{c}$ and $\delta$ the mean value of anomalous dimension $A_{\delta}$ can be equal to 2 , which means that $\frac{v^{2}(\Lambda)}{\Lambda^{2}}$ has an infrared stable quasi fixed-point. If the parameters are in the vicinity of this fixed-point, one can naturally explain the hierarchy $\frac{\bar{v}\left(M_{W}\right)}{\bar{v}\left(M_{P l}\right)} \approx \frac{M_{W}}{M_{P l}}$.

Such an approximate fixed-point behaviour of the parameters in our toy model is expressed in Figs. 1 and 2. We fixed the values of self-interaction coupling and VEV of the scalar field at $174 \mathrm{Gev}$ to be $\bar{\lambda}(174 \mathrm{GEV})=0.25$ and $\bar{v}(174$

8 Note that the strong and nearly equal Yukawa couplings at higher energies provide new explanation for the flavour symmetry breaking in theories with extra dimensions since they are exponentially suppressed at low energies due to the renormalization effects [18]. Quasi fixed-point solutions of high dimensional RG equations in connection with fermion mass hierarchies are thoroughly discussed in [19] 
$\mathrm{GEV})=174 \mathrm{GeV}$ and find that small variation of the initial Yukawa coupling $\bar{h}(174 \mathrm{GEV})=0.55$ (from 0.545 to 0.553 ; this range corresponds to $\bar{h}\left(M_{P l}\right)=$ $2.1 \div 3.5$ ) leads to the very large range of $\bar{v}\left(M_{P l}\right)$ (from $10^{5}$ to $10^{30} \mathrm{GeV}$, see Fig. 1) and $\bar{\lambda}\left(M_{P l}\right)$ (from 0.13 to 0.35 , see Fig. 2) at the Planck scale for the compactification scale $\mu_{c}=10^{16.75} \mathrm{GeV}$ in the case of one extra dimension, $\delta=1$. Thus one can see, while the true fixed-point corresponds to $\bar{\lambda}=\bar{h}=0$, relatively large values of $\bar{\lambda}$ and $\bar{h}$ in the infrared region can be obtained as well starting with the parameters at $M_{P l}$ in a quite large interval. Requiring that $\bar{\lambda}$ and $\bar{h}$ are within the perturbative regime $\left(\frac{\bar{\lambda}^{2}}{4 \pi}<1\right.$ and $\left.\frac{\bar{h}^{2}}{4 \pi}<1\right)$ and that the relations (23a)-(23c) hold for the whole interval between $M_{W}$ and $M_{P l}$, our toy model predicts the upper bounds on the physical masses of the scalar and fermion:

$$
\begin{aligned}
m_{S} & \lesssim 73 \mathrm{GeV} \\
m_{F} & \lesssim 100 \mathrm{GeV} .
\end{aligned}
$$

Finally, we have explicitly cheaked that the ratio $\frac{\bar{v}(\bar{v})}{\bar{v}\left(M_{P l}\right)}$ is actually stable under the variation of the scalar VEV at Planck scale with $\bar{\lambda}\left(M_{P l}\right)$ and $\bar{h}\left(M_{P l}\right)$ fixed. Namely, for $\bar{\lambda}\left(M_{P l}\right)=0.2$ and $\bar{h}\left(M_{P l}\right)=3$ (and for $\mu_{c}=10^{16.75} \mathrm{GeV}, \delta=1$ again) for the average value of the anomalous dimension between the scales $M_{P l}$ and $v$ we obtain

$$
\langle A\rangle \equiv\left(\ln \frac{\bar{v}}{M_{p l}}\right)^{-1} \int_{\ln \frac{\bar{v}}{M_{p l}}}^{0} A_{\delta}(\Lambda) d\left(\ln \frac{\Lambda}{M_{P l}}\right) \approx \frac{2}{1+0.03 \ln \frac{M_{P l}}{\bar{v}\left(M_{P l}\right)}} .
$$

So, if $\bar{v}\left(M_{P l}\right) \approx M_{P l}$, as it is naturally expected, $\langle A\rangle$ is close to 2 , providing large stable hierarchy $\frac{\bar{v}(\bar{v})}{\bar{v}\left(M_{P l}\right)} \approx 1.8 \cdot 10^{-15}$. Thus, varying $\bar{v}\left(M_{P l}\right)$ by $10 \%$ around $10^{17} \mathrm{GeV}$, we obtain $\langle A\rangle \approx 2.14 \div 2.13$ and $\bar{v}(\bar{v})=157 \div 190 \mathrm{GeV}$.

It should be stressed that our solution to the gauge hierarchy problem does not require the extra dimensions to be large. In fact, the hierarchy $\frac{\mu_{c}}{M_{P l}} \sim 0.05 \div 0.3$ is enough to get the desired values of $\bar{v}$ at low energies, even starting with naturally expected large values of $\bar{v}$ at $M_{p l}\left(\bar{v}\left(M_{p l}\right) \sim M_{P l}\right)$. This is demonstrated in Fig. 3, where we have plotted the evolution of $\bar{v}(\Lambda)$ for the case of $\delta=1,2$, and 3 extra dimensions starting from $\bar{v}\left(M_{p l}\right)=10^{17} \mathrm{GeV}$. Requiring that $\bar{v}(174$ $\mathrm{GEV})=174 \mathrm{GeV}$ and $\bar{\lambda}(174 \mathrm{GEV})=0.25, \bar{h}(174 \mathrm{GEV})=0.55$, we obtain $\mu_{c}=10^{16.75}, 10^{17.30}, 10^{17.51} \mathrm{GeV}$ for $\delta=1,2$, and 3 , respectively. For the sake of comparison we have also plotted the running of $\bar{v}(\Lambda)$ in four dimensions $(\delta=0)$. One can see that a familiar fine tuning of parameters at the Planck scale is required in order to achieve the phenomenologically acceptable values of $\bar{v}$ at low energies.

We conclude with the following comments. First, it is clear, that more accurate calculations, related to an improved treatment of thresholds and contributions 
beyond the LPA approximation and truncation (9) of the effective potential as well as higher loop corrections quantitatively alter (perhaps quite significantly) our predictions for particle masses in (25), but qualitatively the behaviour of the parameters seems to remain unchanged, thus providing us with a the natural solution of the gauge hierarchy problem as discussed above. Second, while we have demonstrated our mechanism for the solution of the gauge hierarchy problem on the simplified model of Higgs-Yukawa interaction, we find no reason why it can not work in the realistic models when a full set of the particles and forces of the SM or its extensions will be included. Moreover, several examples of nonsupersymmetric unification through extra dimensions have been recently presented [20], so we believe that a unified model without the gauge hierarchy problem and consistent with present experimental data can be constructed elsewhere.

We would like to thank Z. Berezhiani, J. Chkareuli, G. Dvali, M. Gogberashvili, I. Gogoladze, T. Kobayashi and Z. Tavartkiladze for useful discussions and comments.

\section{References}

[1] N. Arkani-Hamed, S. Dimopoulos and G. Dvali, Phys. Lett. B 429 (1998) 263; I. Antoniadis, N. Arkani-Hamed, S. Dimopoulos and G. Dvali, Phys. Lett. B 436 (1998) 257.

[2] L. Randall and R. Sundrum, Phys. Rev. Lett. 83 (1999) 3370.

[3] M. Gogberashvili, hep-ph/9812296; 9812365; Mod. Phys. Lett. A 14 (1999) 2025, hep-ph/9904383; hep-ph/9908347; L. Randall and R. Sundrum, hepth/9906064; for the generalization to an any number of extra dimensions, see N. Arkani-Hamed, S. Dimopoulos, G. Dvali and N. Kaloper, hep-th/9907209; C. Csaki and Y. Shirman, hep-th/9908186; A.E. Nelson, hep-th/9909001.

[4] J. Lykken and L. Randall, hep-th/9908076; I. Oda, hep-th/9908104; hepth/9909048; H. Hatanaki, M. Sakamoto, M. Tashibana and T. Takenaga, hepth/9909076; A.G. Kohen and D.B. Kaplan, hep-th/9910132.

[5] G.F. Giudice, R. Ratazzi and J.D. Wells, Nucl. Phys. B 544 (1999) 3; E.A. Mirabelli, M. Perelstein and M.E. Peskin, Phys. Rev. Lett. 82 (1999) 2236; T. Han, J.D. Lykken and R.--J. Zhang, Phys. Rev. D 59 (1999) 105006; J.L. Hewett, Phys. Rev. Lett. 82 (1999) 4756.

[6] N. Arkani-Hamed, S. Dimopoulos and G. Dvali, Phys. Rev. D 59 (1999) 086004; S. Cullen and M. Perelstein, hep-ph/9903422; L.J. Hall and D. Smith, hep$\mathrm{ph} / 9904267$.

[7] C. Csaki, M. Graesser, C. Kolda and J. Terning, hep-ph/9906513; J.M. Cline, C. Grojean and G. Servant, hep-ph/9906523; hep-ph/9909496. 
[8] K.R. Dienes, E. Dudas and T. Gherghetta, hep-ph/9908530.

[9] P. Binetruy, C. Deffayet and D. Langlois, hep-th/9905012; N. Kaloper, hepth/9905210: T. Nihei, hep-ph/9905487; D.J. Chung and K. Freese, hepph/9906542; P.J. Steinhardt, hep-th/9907080; W.D. Goldberger and M. Wise, hep-ph/9907218; T. Li, hep-th/9908174; J. Lykken and S. Nandi, hepph/9908505; H.B. Kim and H.D. Kim, hep-th/9909053; U. Elwanger, hepth/9909103; E. Halyo, hep-th/9909127; H. Davoudiasl, J.L. Hewett and T.G. Rizzo, hep-ph/9909255; P. Kanti, I.I. Kogan, K.A. Olive amd M. Pospelov, hep-ph/9909481; J.M. Cline, C. Grojean and G. Servant, hep-ph/9910081; P. Binetruy, C. Deffayet, U. Elwanger and D. Langlois, hep-th/9910219; T. Shiromizu, K.-I. Maeda and M. Sasaki, gr-qc/9910076, E.E. Flanagan, S.-H. H. Tye and I. Wasserman, hep-ph/9910498.

[10] C. Wetterich, Phys. Lett. 104B, 269 (1981); S. Bornholdt and C. Wetterich, Phys. Lett. B282 (1992) 399.

[11] G. Amelino-Camelia, Simple GUTs, in Proc. of the 9th Inter. Sem. Quarks96, Yaroslavl, Russia, 1996; B.C. Allanach, G. Amelino-Camelia and O. Philipsen, Phys. Lett. B393 (1997) 349; B.C. Allanach, G. Amelino-Camelia, O. Philipsen, O. Pisanti and L. Rosa, Nucl.Phys. B537 (1999)32.

[12] K.G. Wilson, Phys. Rev. B4 (1971) 3174, 3184. K.G. Wilson and I.G. Kogut, Phys. Rep. 12C (1974) 75; F.J. Wegner and A. Houghton, Phys. Rev. A8 (1973) 401; J. Polchinski, Nucl. Phys. B231 (1984) 269; A. Ringwald and C. Wetterich, Nucl. Phys. B334 (1990) 506; C. Wetterich, Nucl. Phys. B352 (1991) 529.

[13] T. Kobayashi, J. Kubo, M. Mondragon and G. Zoupanos, Nucl. Phys. B550 (1999) 99; T.E. Clark and S.T. Love, Phys. Rev. D60 (1999) 025005; J. Kubo, H. Terao and G. Zoupanos, hep-ph/9910277.

[14] J.F. Nicoll, T.S. Chang and H.E. Stanley, Phys. Rev. Lett. 33 (1974) 540; A. Hasenfratz and P. Hasenfratz, Nucl. Phys. B270 (1986) 687.

[15] M. Alford, Phys. Lett. B336 (1994) 237; N. Tetradis and C. Wetterich, Nucl. Phys. B442 [FS] (1994) 541; K.-I. Aoki, K.-I. Morikawa, W. Souma, J.-I. Sumi and H. Terao, Prog. Theor. Phys. 95 (1996) 409.

[16] S. Bornholdt and C. Wetterich, Z. Phys. C58 (1993) 585; D.U. Jungnickel and C. Wetterich, Phys. Rev. D53 (1996) 5142.

[17] C. Wetterich in Ref. [12].

[18] K.R. Dienes, E. Dudas and T. Gherghetta, Nucl. Phys. B537 (1999) 47; A.B. Kobakhidze, hep-ph/9904203; K. Yoshioka, hep-ph/9904433.

[19] S.A. Abel and S.F. King, Phys. Rev. D59 (1999) 095010.

[20] K.R. Dienes, E. Dudas and T. Gherghetta in Ref. [18]; P.H. Frampton and A. Rasin, Phys. Lett. B460 (1999) 460; G.K. Leontaris and N.D. Tracas, Phys.Lett. B454 (1999) 53; A. Pérez-Lorenzana and R.N. Mohapatra, hep-ph/9904504. 


\section{Figure captions}

Fig. 1 Fixed-point behaviour of the scalar field VEV in the case of one extra dimension $(\delta=1)$ with the compactification scale $\mu_{c}=10^{16.75} \mathrm{GeV}$.

Fig. 2 Fixed-point behaviour of the scalar field self-interaction coupling $\bar{\lambda}$ in the case of one extra dimension $(\delta=1)$ with the compactification scale $\mu_{c}=$ $10^{16.75} \mathrm{GeV}$.

Fig. 3 The typical running of the scalar field VEV in the case of $\delta=1,2$ and 3 extra dimensions with $\mu_{c}=10^{16.75}, 10^{17.30}, 10^{17.51} \mathrm{GeV}$, respectively. The values of $\operatorname{VEV} \bar{v}\left(M_{P l}\right)=10^{17} \mathrm{GeV}$ and $\bar{v}(174 \mathrm{GeV})=174 \mathrm{GeV}$ are fixed. The running in four dimensions $(\delta=0)$ with $\bar{v}(174 \mathrm{GeV})=174 \mathrm{GeV}$ fixed is also plotted. 


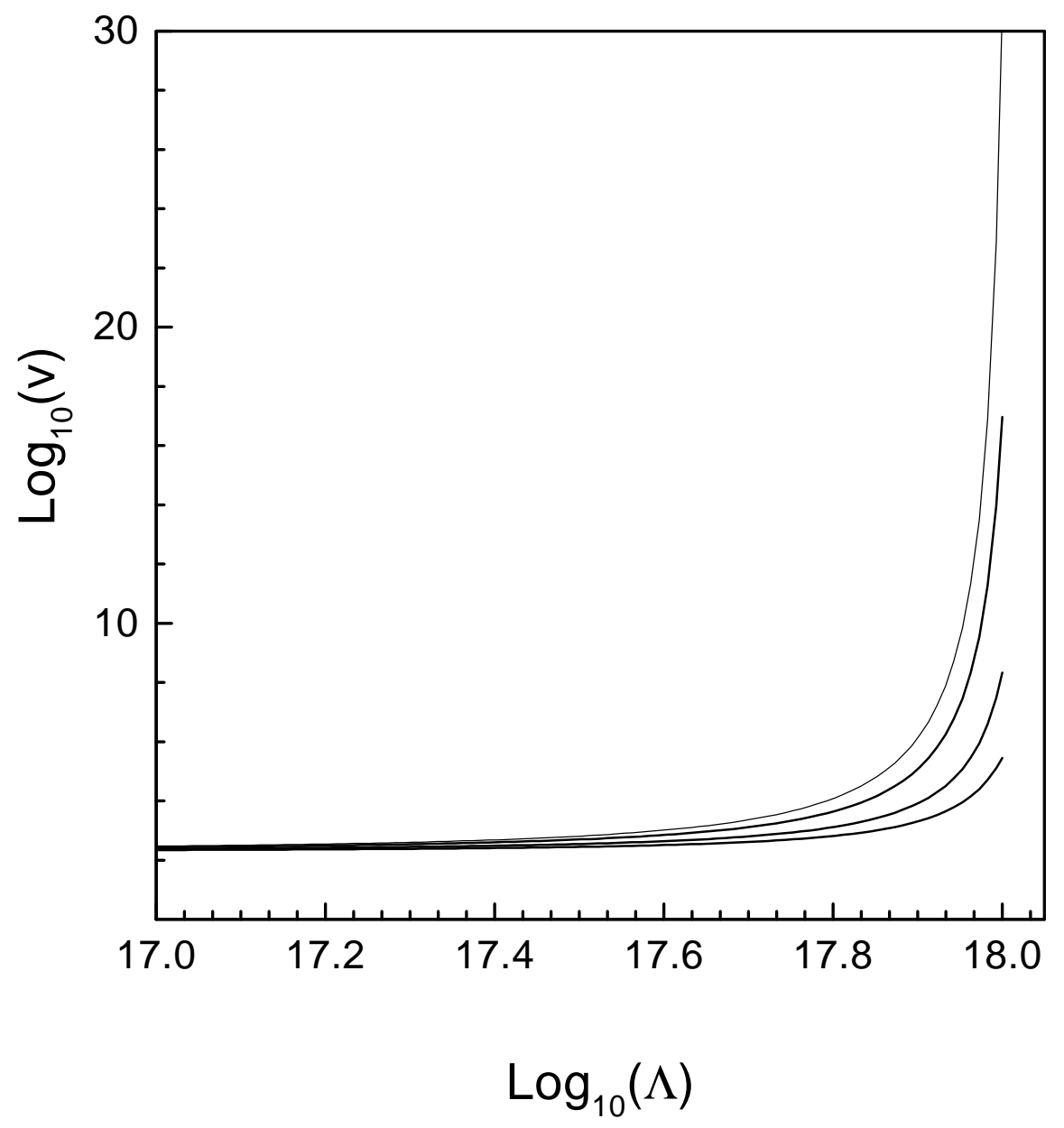

Fig. 1 


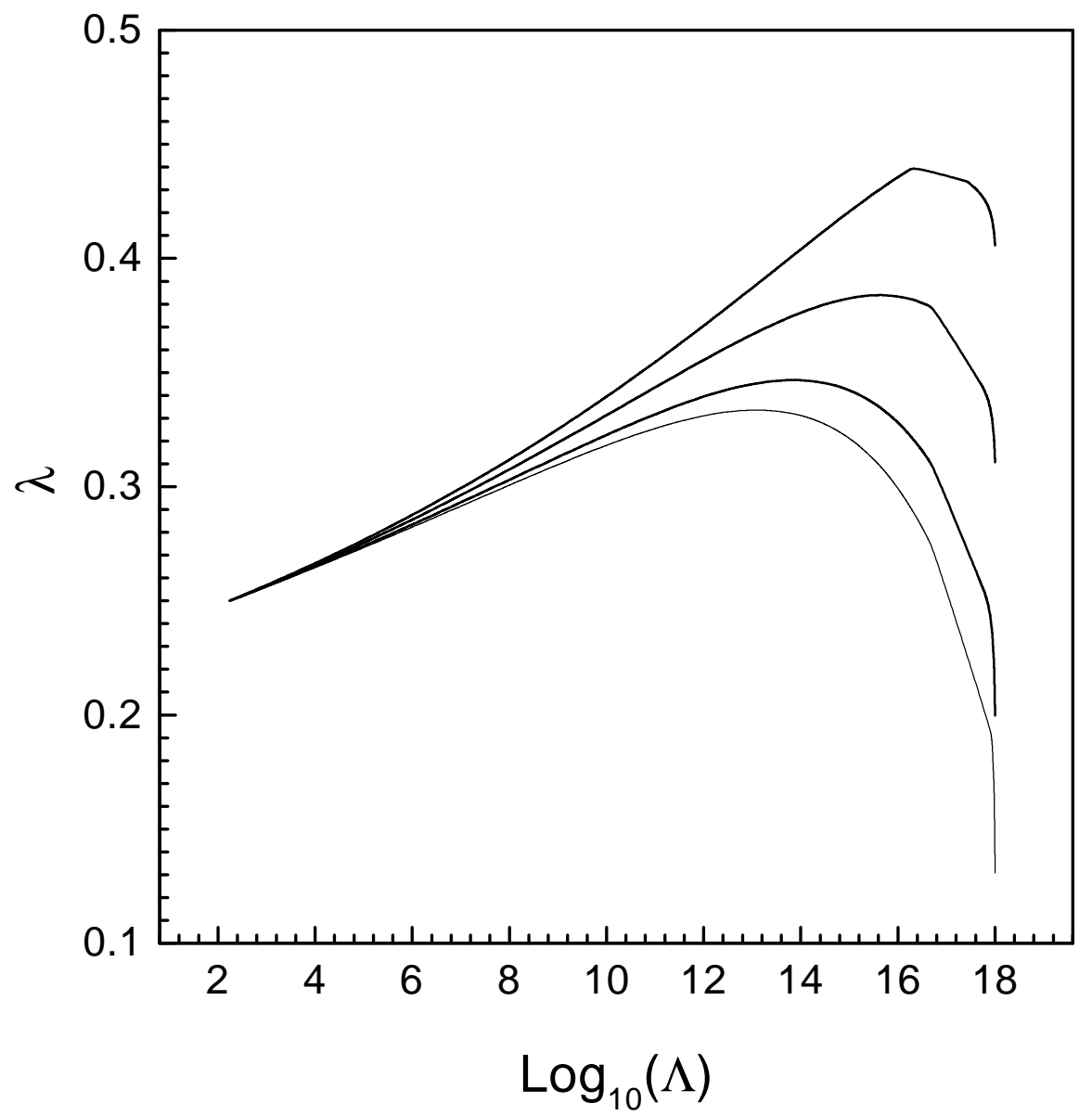

Fig. 2 


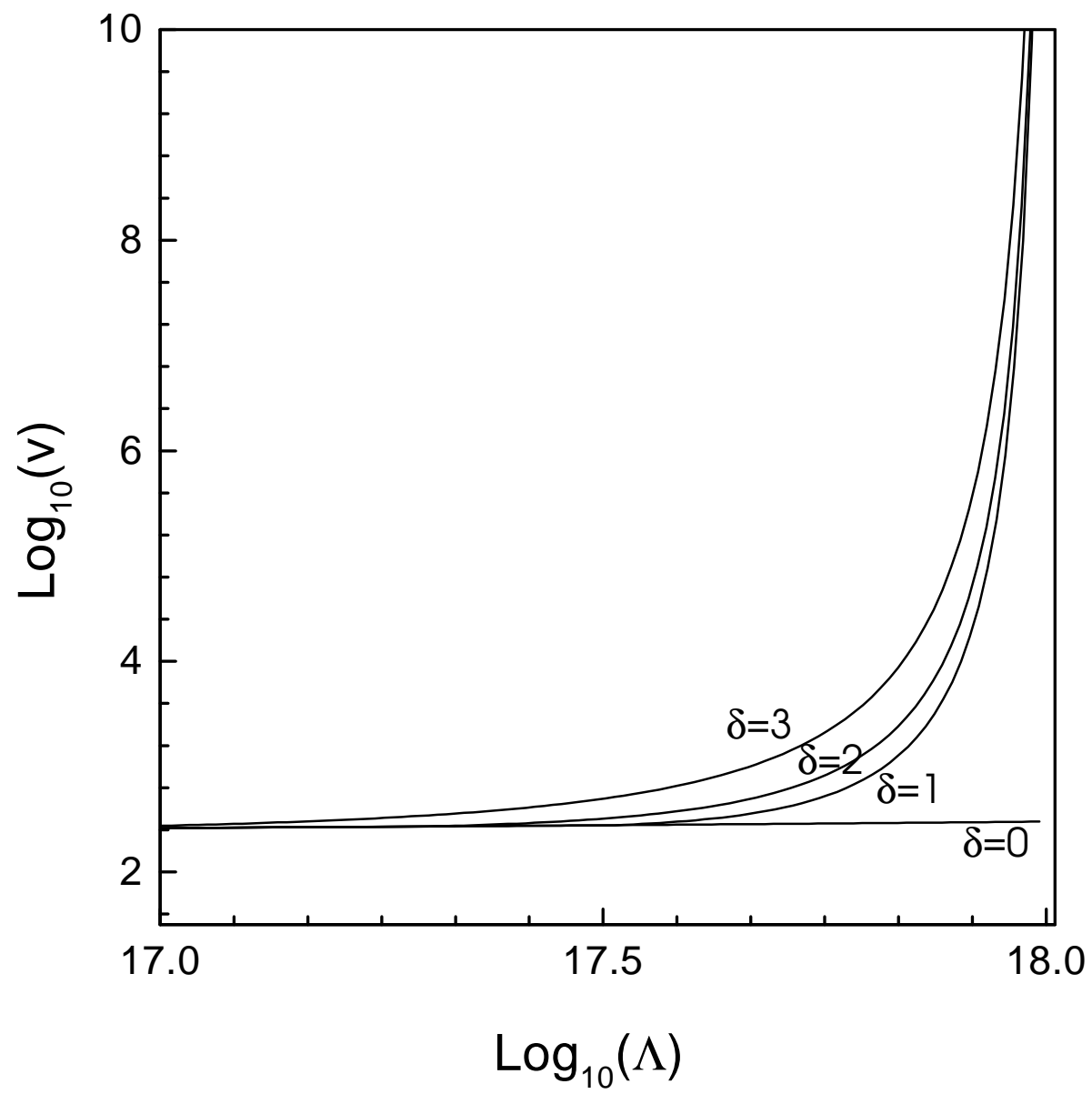

Fig. 3 